\title{
Effective electron-electron interactions and magnetic phase transition in a two-dimensional electron liquid
}

\author{
R. Asgari ${ }^{\mathrm{a}, *}$, A. Esmailian ${ }^{\mathrm{b}}$, B. Tanatar $^{\mathrm{c}}$ \\ ${ }^{a}$ Institute for Studies in Theoretical Physics and Mathematics, Tehran 19395-5531, Iran \\ b Plasma Physics Research Center of Islamic Azad University, Tehran 14835-159, Iran \\ ${ }^{\mathrm{c}}$ Department of Physics, Bilkent University, 06800 Bilkent, Ankara, Turkey
}

Received 26 December 2006; accepted 3 January 2007 by A.H. MacDonald

Available online 10 January 2007

\begin{abstract}
We investigate the spin-dependent effective electron-electron interactions in a uniform system of two-dimensional electrons to understand the spontaneous magnetization expected to occur at very low density. For this purpose, we adopt the Kukkonen-Overhauser form for the effective interactions which are built by accurately determined local-field factors describing the charge and spin fluctuations. The critical behavior of the effective interaction for parallel spin electrons allows us to quantitatively locate the transition to the ferromagnetic state at $r_{s} \approx 27$. When the finite width effects are approximately taken into account the transition occurs at $r_{s} \approx 30$ in agreement with recent quantum Monte Carlo calculations. (C) 2007 Elsevier Ltd. All rights reserved.
\end{abstract}

PACS: 71.10.Ca; 05.30.Fk

Keywords: D. Electron-electron interactions; D. Magnetic phase transition

\section{Introduction}

Electron-electron interactions are known to produce the most pronounced effects in two-dimensional (2D) electron systems as numerous experimental and theoretical investigations reveal [1,2]. As the fabrication techniques continue to improve, it has been possible to probe the low density regime of $2 \mathrm{D}$ electrons where strong interaction effects become more significant. In the last decade or so, a large amount of effort concentrated on the low density and low temperature ground state properties of 2D electron systems with a view to understanding the metal-insulator transition and its possible mechanisms [3]. The emergent picture indicates the important role played by strong interaction effects.

In connection with the metal-insulator transition, the spin susceptibility of 2D electrons has also caught attention because of a possible magnetic phase transition in the same density

\footnotetext{
* Corresponding address: Department of Physics, Institute for Studies in Theoretical Physics and Mathematics, Tehran 19395-5531, Iran. Tel.: +98 21 222 80692; fax: +98 2122280415.

E-mail address: asgari@theory.ipm.ac.ir (R. Asgari).
}

regime. Whereas recent experiments are performed under an in-plane magnetic field to spin polarize the electrons, a more fundamental problem is to determine whether the system spontaneously magnetizes at some critical density. At low densities the electron-electron interactions dominate over the kinetic energy and the 2D electron system becomes unstable to spin polarization. A few experiments $[4,5]$ have indicated the observation of such a possibility.

Theoretically, the spontaneous magnetization of a 2D electron liquid has been considered in a variety of many-body approaches [6-8]. These calculations mostly depend on the accurate evaluation of the ground-state energy for paramagnetic and ferromagnetic phases. The most accurate results are obtained from quantum Monte Carlo (QMC) simulations [911] which predict a first order transition from a spin unpolarized state to a fully spin polarized state around $r_{s} \approx 25.5$, where the dimensionless parameter $r_{s}$ is the ratio of the interaction energy to the kinetic energy. This transition is followed by a liquid to Wigner solid transition [11,12] at $r_{s} \approx 37$. As an alternative approach Davoudi et al. [13] considered the behavior of spin-dependent effective electron-electron interactions to study the magnetic phase transition. As the transition to the 
ferromagnetic state is approached the effective interactions between like spins become negative at long wavelength and at the critical density corresponding to $r_{\mathrm{sc}}$ suddenly change sign. The signature for such a behavior is ultimately related to the long wavelength limit of the spin susceptibility which can be regarded as an order parameter.

The purpose of this paper is to investigate quantitatively the transition density to a ferromagnetic state in a paramagnetic 2D electron gas using accurately constructed effective electron-electron interactions. We adopt the Kukkonen-Overhauser [14] form of the effective interactions and use recently developed local-field factors [15] to calculate their wave vector and density dependence. The spinsymmetric and antisymmetric local-field factors have been obtained from the accurate QMC simulations [16] via the fluctuation-dissipation theorem. Our numerical calculations of the long wavelength behavior of effective electron-electron interactions for the parallel and anti-parallel spins allow us to quantitatively predict the paramagnetic to ferromagnetic phase transition to occur at $r_{\mathrm{sc}} \approx 27$. Extending our calculations to a finite quantum well we find that the same transition takes place at $r_{\mathrm{sc}} \approx 30$. These findings are in good agreement with other theoretical approaches using ground state energy methods.

The rest of this paper is organized as follows. In the next section we present the expressions for the spin-dependent effective interactions for 2D electron systems. We describe briefly the way we obtain the local-field factors describing the charge and spin-density correlations which constitute the crucial input for effective interactions. Our numerical results are presented in Section 3, followed by a short summary.

\section{Theory}

We consider both 2D and Q2D electron liquids as models for systems of electronic carriers with band mass $m$ in a semiconductor heterojunction with dielectric constant $\kappa$. We include the effect of thickness of a GaAs heterojunctioninsulated gate field-effect transistor (HIGFET) with bare electron-electron interaction $v_{\mathbf{q}}=2 \pi e^{2} F(q d) /(\kappa q)$ which is the Coulomb potential renormalized by the form factor given by

$F(x)=\left(1+\frac{\kappa_{\mathrm{ins}}}{\kappa_{\mathrm{sc}}}\right) \frac{8+9 x+3 x^{2}}{16(1+x)^{3}}+\left(1-\frac{\kappa_{\mathrm{ins}}}{\kappa_{\mathrm{sc}}}\right) \frac{1}{2(1+x)^{6}}$,

where $d=\left[\hbar \kappa_{\mathrm{sc}} /\left(48 \pi m e^{2} n^{*}\right)\right]^{1 / 3}$ and $n^{*}=n_{\mathrm{depl}}+11 n / 32$. Here the depletion layer charge density $n_{\text {depl }}$ being essentially zero and $\kappa_{\text {ins }}=10.9$ and $\kappa_{\text {sc }}=12.9$ and $\kappa$ is their average. At zero temperature there are only two relevant parameters for a disorder-free, homogeneous 2D electron liquid in the absence of quantizing magnetic fields, the usual Wigner-Seitz density parameter $r_{s}=\left(\pi n a_{B}^{2}\right)^{-1 / 2}, a_{B}=\hbar^{2} \kappa /\left(m e^{2}\right)$ being the Bohr radius in the medium of interest and the second parameter is the degree of spin polarization $\zeta=\left|n_{\uparrow}-n_{\downarrow}\right| / n$. Here $n_{\sigma}$ is the average density of particles with spin $\sigma=\uparrow, \downarrow$ and $n=n_{\uparrow}+n_{\downarrow}$ is the total average density. Evidently, when the form factor
$F(x)=1$, we recover the strictly $2 \mathrm{D}$ electron liquid bare potential.

In this paper we are concerned with the effective electron-electron interactions in the paramagnetic phase, $\zeta=$ 0 , of an electron system as functions of the coupling strength, $r_{s}$. The problem of calculating the effective electron-electron interactions, or generally, between two quasiparticles in a degenerate Fermi liquid has been addressed by several authors $[14,17,18]$. The simple random phase approximation (RPA) for the effective potentials leads to $v_{\mathbf{q}} / \epsilon(q, \omega)$, where $\epsilon(q, \omega)$ is the RPA dielectric function. RPA has been very successful in describing the dielectric properties of interacting quantum liquids in the high density limit. As the density of the system is lowered, the exchange and correlation effects become very important. Kukkonen and Overhauser [14] derived an expression which accounts for the charge and spin fluctuations and particular attention was paid to the indistinguishability of the electrons in the system. The spin-symmetric and spinantisymmetric local-field factors entering these expressions are to be determined by independent methods. Later, Vignale and Singwi [18], using diagrammatic techniques, derived a similar expression given in terms of polarization fields.

Following the Kukkonen and Overhauser approach [14], the effective electron-electron interaction between two electrons with spin indices $\sigma$ and $\sigma^{\prime}$ is written in momentum space as

$$
\begin{aligned}
V_{\sigma \sigma^{\prime}}(q, \omega)= & v_{\mathbf{q}}+\left\{v_{\mathbf{q}}\left[1-G_{s}(q, \omega)\right]\right\}^{2} \chi_{s}(q, \omega) \\
& +\vec{\sigma} \cdot \overrightarrow{\sigma^{\prime}}\left\{v_{\mathbf{q}} G_{a}(q, \omega)\right\}^{2} \chi_{a}(q, \omega) .
\end{aligned}
$$

The first term is the bare interaction between two electrons. The second and the third terms are interactions mediated by charge and spin-density fluctuations, respectively. The dielectric properties of electron liquids are described by the density-density response function

$\chi_{s}(q, \omega)=\frac{\chi_{0}(q, \omega)}{1-v_{\mathbf{q}}\left[1-G_{s}(q, \omega)\right] \chi_{0}(q, \omega)}$,

and spin-spin response function

$\chi_{a}(q, \omega)=\frac{\chi_{0}(q, \omega)}{1+v_{\mathbf{q}} G_{a}(q, \omega) \chi_{0}(q, \omega)}$,

where $\chi_{0}(q, \omega)$ is the non-interacting response function, i.e. the Lindhard function, which contains the non-interacting occupation numbers [2]. The wave vector and frequency dependent local-field factors $G_{s}(q, \omega)$ and $G_{a}(q, \omega)$ describe the short-range electron correlations in the spin-symmetric and spin-antisymmetric channels. To calculate the screening contribution of effective electron-electron interactions, we only need to focus on the static limit by considering $\omega=0$ in Eq. (2).

\section{Numerical results}

As is clear from Eq. (2) the local-field factors are the fundamental quantities for an evaluation of effective electron-electron interactions. In this section we introduce the static values of these functions. Our strategy follows that of our recent work [15] which uses accurate spin-symmetric and 


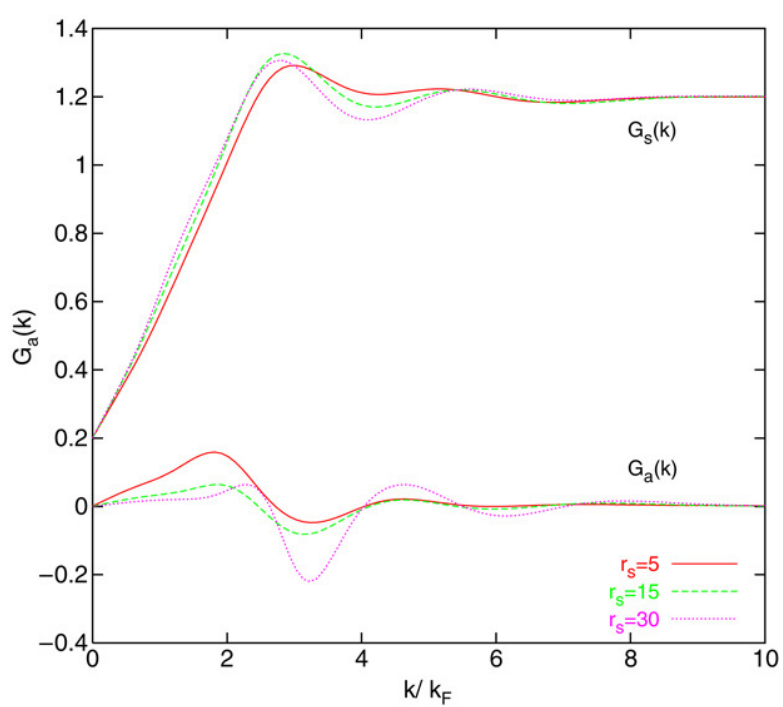

Fig. 1. (Color online) The spin-symmetric and spin-antisymmetric local-field factors, $G_{S}(k)$ and $G_{a}(k)$ as functions of $k / k_{F}$ in the $2 \mathrm{D}$ electron liquid at various values of $r_{s}$.

spin-antisymmetric static structure factors to build the localfield factors. For this purpose, we have calculated the spinsymmetric and spin-antisymmetric pair distribution functions from the quantum Monte Carlo (QMC) based analytical expressions [16] and performed the Fourier transform

$S_{s, a}(q)=1+n \int \mathrm{d} \mathbf{r}\left[g_{s, a}(r)-1\right] \exp (-\mathbf{i q} \cdot \mathbf{r})$.

The structure factors $S_{s, a}(q)$ are assured to satisfy the plasmon and f-sum rules. Finally, within the fluctuation-dissipation theorem, which is given by

$S_{s, a}(q)=-\frac{1}{n \pi} \int_{0}^{\infty} \mathrm{d} \omega \Im\left[\chi_{s, a}(q, \omega)\right]$,

where $\chi_{s, a}(q, \omega)$ depend on $G_{s, a}(q)$, one may determine the local-field factors once the static structure factors are given. It is necessary to point out that the long-wavelength limit of $G_{s}(q)$ is not recovered and we thus correct our $G_{s}(q)$ using the longwavelength form of the polarization potential calculation which is constructed to satisfy this limit [15]. The main results of our work are shown in Figs. 1-5.

We start by showing in Fig. 1 the spin-symmetric and antisymmetric local-field factors at $r_{s}=5,15$ and 30. One of the important features of the present local-field factors is their oscillatory behavior. The first peaks of $G_{s}(k)$ and $G_{a}(k)$ around $3 k_{F}$ and $2 k_{F}$, respectively, are corroborated by other calculations $[19,20]$.

In Fig. 2 we show the effective electron-electron interaction $V_{\uparrow \downarrow}(k)$ for antiparallel-spins in both strictly 2D (thin lines) and Q2D (thick lines) electron liquids in the paramagnetic state at $r_{s}=5,10,20$ and 25. Here the effect of quantum well thickness is included through the form factor and the localfield factors are calculated for a strictly 2D electron liquid through the fluctuation-dissipation theorem for both cases. This is an approximation in the absence of QMC data for Q2D systems. It is clear from this figure that including the

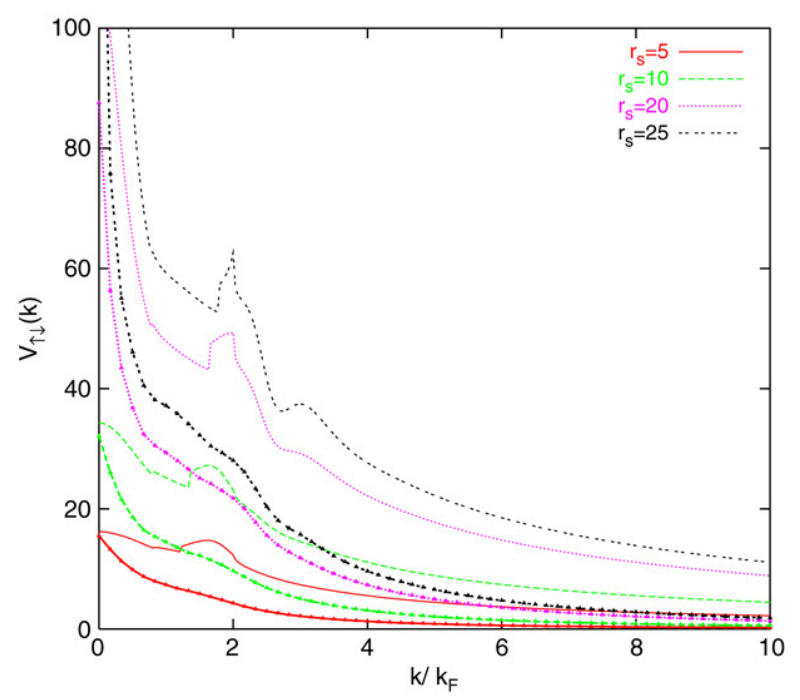

Fig. 2. (Color online) Effective electron-electron interaction potential in momentum space for antiparallel-spin electrons in the 2D (thin lines) and Q2D (thick lines) electron liquid at various values of $r_{S}$

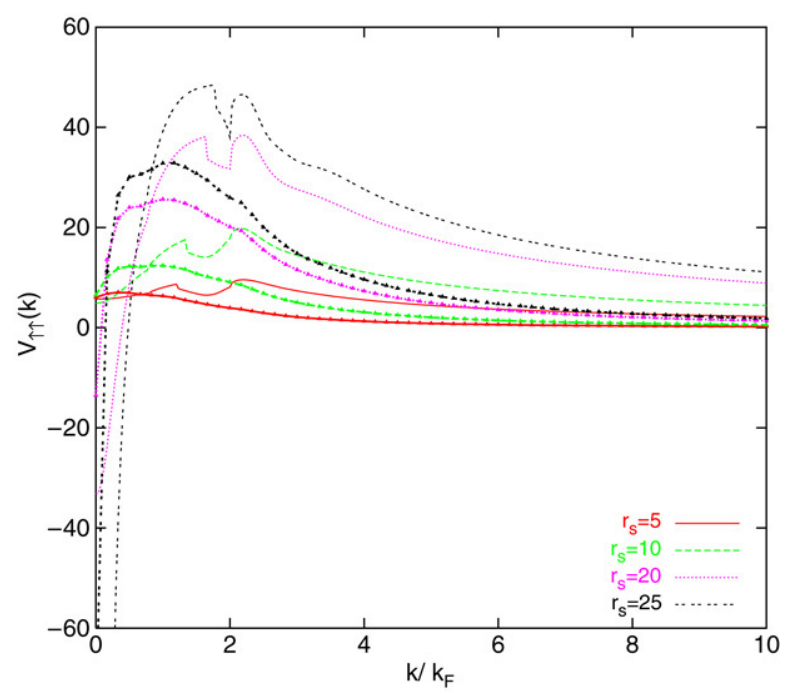

Fig. 3. (Color online) Effective electron-electron interaction potential in momentum space for parallel-spin electrons in the 2D (thin lines) and Q2D (thick lines) electron liquid at various values of $r_{S}$.

form factor reduces the value of the effective potential and makes it shorter ranged, as $V_{\uparrow \downarrow}(k)$ decays at large $k$ faster than $1 / k$. In contrast to the calculations of Davoudi et al. [13] which show a flat behavior for $k \leq 2 k_{F}$, our antiparallel-spin potential $V_{\uparrow \downarrow}(k)$ is a smooth function in the long-wavelength region. A peak near $k=2 k_{F}$ develops with increasing $r_{s}$ due to the structure of $\chi_{0}\left(2 k_{F}, 0\right)$ corresponding to Friedel oscillations in coordinate space. Furthermore, by increasing the $r_{s}$ value, $V_{\uparrow \downarrow}(0)$ at contact increases and shows strong repulsive interactions between two electrons with opposite spins.

Fig. 3 displays the effective electron-electron interaction $V_{\uparrow \uparrow}(k)$ for parallel-spins in both strictly 2D (thin lines) and Q2D (thick lines) electron liquids in the paramagnetic state at $r_{s}=5,10,20$ and 25. Again, in contrast to the results of Davoudi et al. [13], $V_{\uparrow \uparrow}(k)$ is a smooth function in the long- 


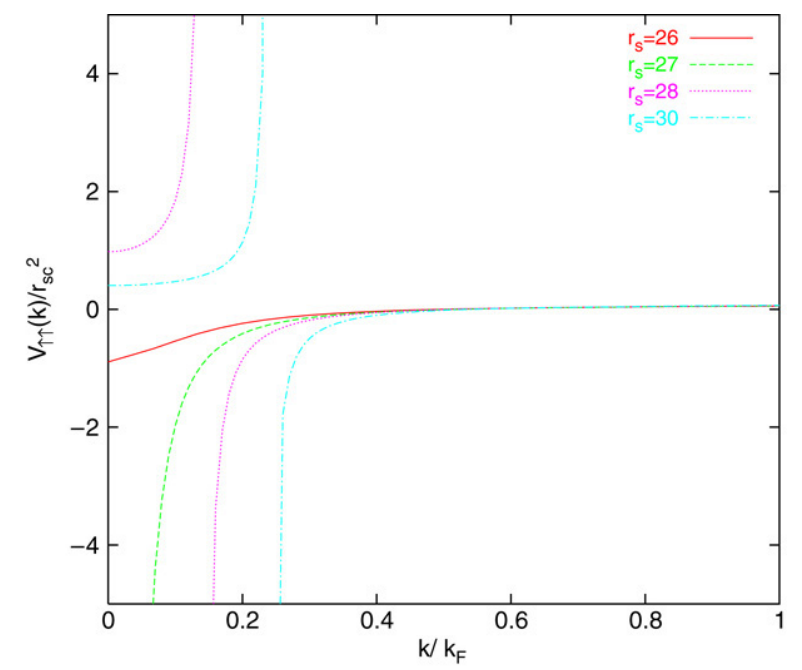

Fig. 4. (Color online) Long-wavelength behavior of effective electron-electron interaction potential divided by $r_{\mathrm{sc}}^{2}=(27)^{2}$ (for illustration purposes) in momentum space for parallel-spin electrons in the 2D electron liquid near the critical $r_{s}$ value.

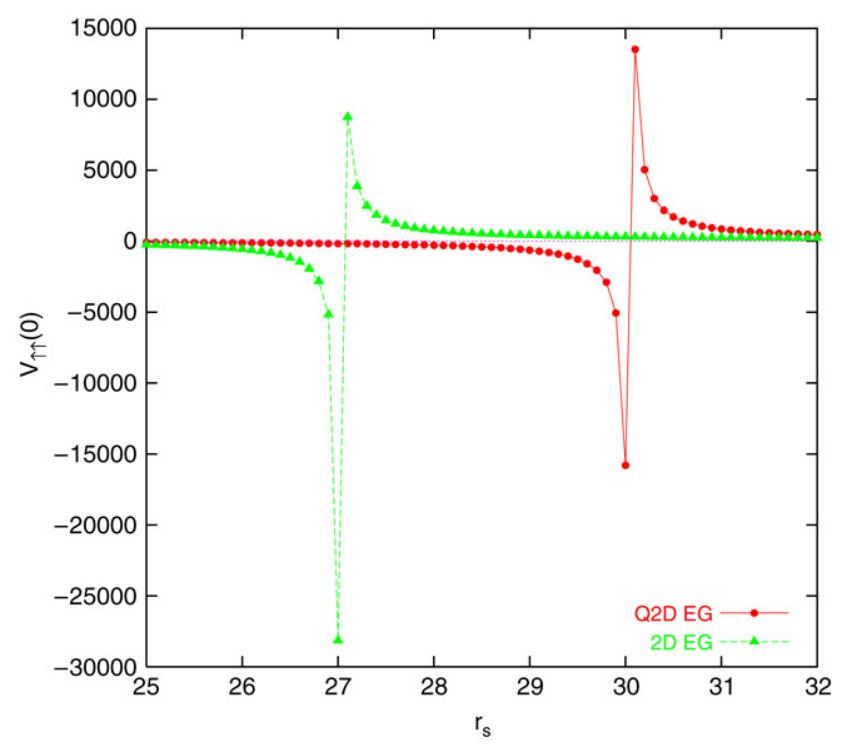

Fig. 5. (Color online) Effective electron-electron interaction potential with parallel-spins at contact as a function of $r_{S}$ in the $2 \mathrm{D}$ electron liquid with $r_{\mathrm{Sc}} \approx 27$ and Q2D electron liquid with $r_{\mathrm{Sc}} \approx 30$.

wavelength region and a peak develops around $k=2 k_{F}$ with increasing $r_{s}$ due to the structure of $\chi_{0}\left(2 k_{F}, 0\right)$. Furthermore, by increasing the $r_{s}$ value, $V_{\uparrow \uparrow}(0)$ at contact becomes more negative and shows strong attractive interactions between two electrons with parallel-spins. This is a signal for the magnetic phase transition which we would like to focus on. This behavior has been anticipated and reported by Davoudi et al. [13] by constructing the local-field factors at large $r_{s}$ values up to $r_{s}=$ 20 qualitatively. Here we have the quantitative details of the effective electron-electron interactions by having an accurate evaluation of $\chi_{s, a}(q)$.

We present in Fig. 4 the effective electron-electron interaction for parallel spins divided by $r_{\mathrm{sc}}^{2}=(27)^{2}$ (for illustration purposes) in the $2 \mathrm{D}$ electron liquid in the vicinity of the magnetic phase transition, at $r_{s}=26,27,28$ and 30. It is evident from these results that the value of $V_{\uparrow \uparrow}(0)$ displays a critical behavior at $r_{\mathrm{sc}}$. Being negative for $r_{s}<$ $r_{\mathrm{sc}}$, the effective electron-electron interaction at $k=0$ for parallel-spins regains a positive value after the magnetic phase transition takes place. In other words, the sign change of $V_{\uparrow \uparrow}(0)$ indicates the magnetic phase transition. We have also found that for $r_{s}>r_{\mathrm{sc}}, V_{\uparrow \uparrow}(k)$ exhibits a singularity at some finite $k$, indicating the breakdown of the paramagnetic formulation of the Kukkonen-Overhauser effective interaction in the ferromagnetic phase.

To calculate the magnetic phase transition more quantitatively, we have shown $V_{\uparrow \uparrow}(0)$ as a function of $r_{s}$ both for 2D and Q2D electron liquids in Fig. 5. From the physical interpretation discussed above this figure shows that there is a magnetic phase transition for the $2 \mathrm{D}$ electron liquid at $r_{\mathrm{sc}}=27$ which is very close to the one predicted by QMC simulations [11] at $r_{s}=25.5$ and another theoretical calculation based on the Fermi-hypernetted chain approximation [21] at $r_{s}=24$. When the finite width effects are included approximately, we find a transition from paramagnetic to the fully spin-polarized fluid for the Q2D electron liquid at $r_{\mathrm{sc}}=30$ which is very close to the QMC result of De Palo et al. [22], $r_{s} \simeq 27$. Such good agreement with other calculations on the one hand verifies the importance of effective interactions in the strongly coupled regime of $2 \mathrm{D}$ electron systems. Furthermore, it also implies that the local-field factors we have developed are consistent with the ground state energy values obtained in QMC simulations.

\section{Summary}

In summary, we have addressed the problem of the paramagnetic to ferromagnetic phase transition in $2 \mathrm{D}$ electron systems expected to occur at very low density. We have constructed the spin dependent effective electron-electron interactions using accurate local-field factors derived from QMC simulations and the fluctuation-dissipation theorem. The long wavelength limit of the effective interaction for parallel spin electrons exhibits a critical behavior approaching the magnetic phase transition. Our results for the critical densities at which such a transition occurs for 2D and Q2D systems are in good agreement with other theoretical calculations employing ground state energy comparisons in different phases.

\section{Acknowledgments}

This work is supported by TUBITAK and TUBA.

\section{References}

[1] T. Ando, A.B. Fowler, F. Stern, Rev. Modern Phys. 54 (1982) 437.

[2] G.F. Giuliani, G. Vignale, Quantum Theory of the Electron Liquid, Cambridge University Press, Cambridge, England, 2005.

[3] E. Abrahams, S.V. Kravchenko, M.P. Sarachik, Rev. Modern Phys. 73 (2001) 251;

S.V. Kravchenko, M.P. Sarachik, Rep. Progr. Phys. 67 (2004) 1.

[4] A. Ghosh, C.J.B. Ford, M. Pepper, H.E. Beere, D.A. Ritchie, Phys. Rev. Lett. 92 (2004) 116601. 
[5] R. Winkler, E. Tutuc, S.J. Papadakis, S. Melinte, M. Shayegan,

D. Wasserman, S.A. Lyon, Phys. Rev. B 72 (2005) 195321.

[6] A.K. Rajagopal, J.C. Kimball, Phys. Rev. B 15 (1977) 2819.

[7] B. Davoudi, M.P. Tosi, Physica B 322 (2002) 124.

[8] Y. Zhang, S. Das Sarma, Phys. Rev. B 72 (2005) 115317.

[9] D. Varsano, S. Moroni, G. Senatore, Europhys. Lett. 53 (2001) 348.

[10] G. Senatore, S. Moroni, D. Varsano, Solid State Commun. 119 (2001) 333.

[11] C. Attaccalite, S. Moroni, P. Gori-Giorgi, G.B. Bachelet, Phys. Rev. Lett. 88 (2002) 256601.

[12] B. Tanatar, D.M. Ceperley, Phys. Rev. B 39 (1989) 5005.

[13] B. Davoudi, M. Polini, G. Sica, M.P. Tosi, Solid State Commun. 121 (2002) 295.
[14] C.A. Kukkonen, A.W. Overhauser, Phys. Rev. B 20 (1979) 550.

[15] R. Asgari, A.L. Subaş1, A.A. Sabouri-Dodaran, B. Tanatar, Phys. Rev. B 74 (2006) 155319.

[16] P. Gori-Giorgi, S. Moroni, G.B. Bachelet, Phys. Rev. B 70 (2004) 115102.

[17] C.F. Richardson, N.W. Ashcroft, Phys. Rev. B 55 (1997) 15130.

[18] G. Vignale, K.S. Singwi, Phys. Rev. B 31 (1985) 2729; 32 (1985) 2156.

[19] G.S. Atwal, I.G. Khalil, N.W. Ashcroft, Phys. Rev. B 67 (2003) 115107.

[20] M.W.C. Dharma-wardana, F. Perrot, Europhys. Lett. 63 (2003) 660.

[21] R. Asgari, B. Davoudi, M.P. Tosi, Solid State Commun. 131 (2004) 130.

[22] S. De Palo, M. Botti, S. Moroni, G. Senatore, Phys. Rev. Lett. 94 (2005) 226405. 